Document downloaded from:

http://hdl.handle.net/10251/149639

This paper must be cited as:

Ortigosa, N.; Galbis Verdu, A.; Fernández, C.; Cano-Pérez, O. (2017). Gabor frames for classification of paroxysmal and persistent atrial fibrillation episodes. Medical Engineering \& Physics. 39:31-37. https://doi.org/10.1016/j.medengphy.2016.10.013

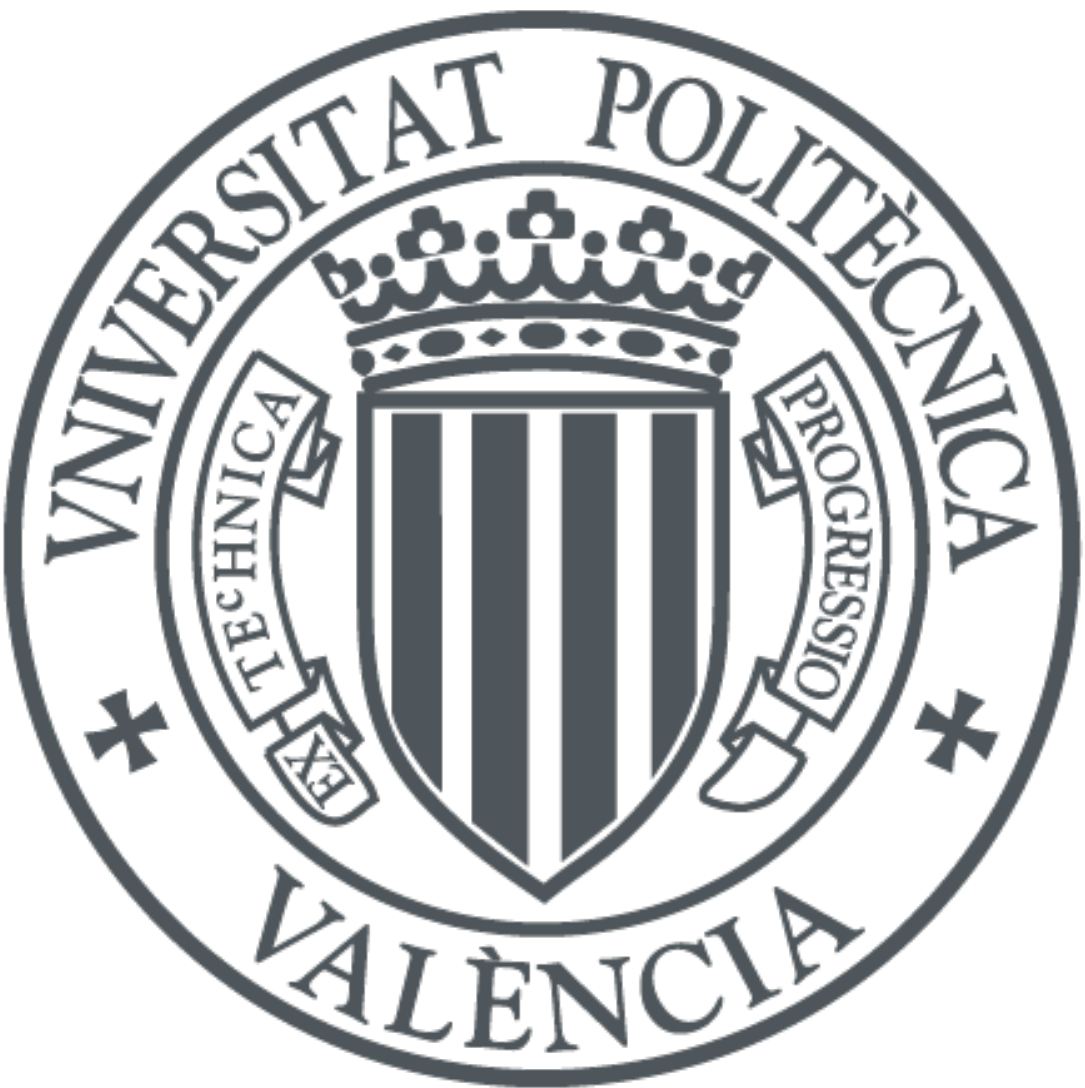

The final publication is available at

https://doi.org/10.1016/j.medengphy.2016.10.013

Copyright Elsevier

Additional Information 
1 Word count from abstract to the end of the acknowledgements

2 section: 4093 words 


\title{
Gabor frames for classification of paroxysmal and persistent atrial fibrillation episodes
}

\author{
Nuria Ortigosa $^{\mathrm{a}}$, Antonio Galbis ${ }^{\mathrm{b}}$, Carmen Fernández $^{\mathrm{b}}$, Óscar Cano ${ }^{\mathrm{c}}$ \\ ${ }^{a}$ I.U. Matemática Pura y Aplicada, Universitat Politècnica de València \\ Camino de Vera s/n, 46022 Valencia, Spain \\ nuorar@upvnet.upv.es \\ ${ }^{b}$ Univ. Valencia, Dept. Math. Analysis, E-46100 Burjassot, Spain \\ antonio.galbis@uv.es,fernand@uv.es \\ ${ }^{c}$ Hospital Universitari i Politècnic La Fe \\ Servicio de Cardiología, Instituto de Investigación Sanitaria La Fe \\ Planta 4-Torre F, Av. Bulevar Sur s/n, 46026 Valencia, Spain \\ cano_osc@gva.es
}

\section{Abstract}

In this paper, we propose a new classification method for early differentiation of paroxysmal and persistent atrial fibrillation episodes, i.e. those which spontaneously or with external intervention will return to sinus rhythm within 7 days of onset from the ones where the arrhythmia is sustained for more than 7 days. Today, clinicians provide patients classification once the course of the arrhythmia has been disclosed. In this work we deal with this problem studying a sparse representation of surface electrocardiogram signals by means of Gabor frames and applying a linear discriminant analysis afterwards. Thus, we provide an early discrimination, obtaining promising performances on a heterogeneous cohort of patients in terms of pharmacological treatment and state of progression of the arrhythmia: 95\% sensitivity, $82 \%$ specificity, $89 \%$ accuracy. In this manner, the proposed method can 
help clinicians to choose the most appropriate treatment using the electrocardiogram, which is a widely available and non-invasive technique. This early differentiation is clinically highly significant in order to choose optimal patients who may undergo catheter ablation with higher success rates.

Keywords: Gabor frames, Atrial Fibrillation, Electrocardiogram

\section{Introduction}

Atrial fibrillation (AF) is the most common cardiac arrhythmia in clinical practice, and affects up to 4.5 million people in Europe and 2.3 million adults in USA. Its prevalence increases with age, being less than $1 \%$ among adults younger than 60 years but about $9 \%$ for people older than 80 . Indeed, it is likely to increase 2.5-fold by the year 2050 [1].

$\mathrm{AF}$ is a supraventricular arrhythmia characterized by uncoordinated atrial activation and ineffective atrial contraction, which is reflected on the ECG by irregular heart beat intervals and absence of P-wave [2]. Clinical practice guidelines to manage patients with AF classify them by the duration of the AF episodes as paroxysmal (episodes which spontaneously or with external intervention return to sinus rhythm within seven days after their onset), persistent (patients in whom AF is sustained more than seven days and require pharmacological or electrical cardioversion to restore sinus rhythm), and permanent (both the patient and clinician accept to stop further attempts to control rhythm) $[3,4,5]$.

Many references of the state-of-the-art have performed an analysis and 
classification of AF episodes, most of which have used the public AF termination database of Physionet [6], that consists of one-minute ECGs of sustained or self-terminating AF after one second, one minute, or at least one hour of the end of the record.

Thus, classification of AF episodes and their spontaneous termination by means of the surface ECG has been addressed in several works, mainly by means of the analysis of the dominant frequency of the atrial activity (AA) signal: by observing more likelihood to terminate AF when the dominant frequency decreases [7] and also to characterize the circadian rhythms of persistent atrial fibrillation [8]. Other references have used the modulus and phase information of several time-frequency transforms $[9,10]$ to perform the AF paroxysmal and persistent classification, or hidden Markov models to track the frequency changes along ECG signals to early detect AF episodes nearly to terminate [11]. Other authors also study other features, apart from the dominant atrial frequency, such as the amplitude and the waveform shape of the AA [12] or the average heart rate and the index of ventricular activity [13] as optimal discriminators between self-terminating and sustained episodes along 24 hours.

Non-linear measures, such as sample entropy, have also been used to observe differences between the AF episodes analyzing the main atrial wave [14], and using long-term ECG recordings $[15,16]$, or even intracardiac recordings $[17]$.

Regrettably, although manifold tools have been developed to aid clini- 
cal decision recently, the validation across the broad range of AF patients is still incomplete [18]. In this paper we deal with the early discrimination of paroxysmal and persistent AF episodes classified according to current clinical guidelines $[3,4,5]$. We perform this clinical classification addressed in previous papers $[9,10]$ from a different point of view. We have extracted the coefficients of a sparse representation with respect to a Gabor frame, which have been calculated from the frequency spectrum information of the ECG signal, once ventricular activity has been canceled. Then, we use linear discriminant analysis for classification.

Sparse representation of signals has been recently introduced for biomedical signal analysis. In particular, for ECG processing, it has been mainly used for signal compression [19, 20, 21] and beat classification [22]. Particularly, sparse dictionaries have been applied for ventricular and atrial activity estimation in patients suffering from AF in the works presented in [23, 24]. In this paper, we have applied sparse representation by means of Gabor frames on a cohort of signals acquired from real patients, providing good classification results. Moreover, one value-add of the current work is the diversity of patients included in the cohort under study, in terms of antiarrhythmic treatments and state of progression of the arrhythmia.

The rest of the paper is organized as follows. The study population and its clinical characteristics are described in Section 2. Signal pre-processing and the feature extraction methods by means of the sparse representation are described in Sections 3.1-3.4. Next, experimental results and performances 
96

are depicted in Section 4. Finally, discussion of results is presented in Section 5 and conclusions are drawn in Section 6.

\section{Materials}

The population of this retrospective study consists of 186 consecutive unselected patients who were suffering from paroxysmal or persistent atrial fibrillation. They were attended in a specific arrhythmia clinic of a tertiary center (La Fe Hospital, Valencia), where the bipolar lead II was registered for five seconds and stored in PDF format by using the Philips PageWriter TC50 electrocardiograph. Corresponding original raw data was extracted from the PDF file by using the application presented in [25]. Lead II was analysed since it is the rhythm strip regularly registered by default in this tertiary centre, due to the easy visualization of the presence/absence of P-waves.

There were 41 paroxysmal and 145 persistent patients, whose AF categorization was defined according to the current guidelines [3, 4, 5]. This cohort results in a study different from the several references which have studied the AF termination and differentiation based on the Physionet AF Termination Database [6] and Long-Term AF Database [7], which consider paroxysmal patients (as those with self-terminating short episodes) and sustained AF when it lasts for more than 24 hours, most of which correspond to permanent $\mathrm{AF}$.

Furthermore, as the number of subjects included in each group is clearly unbalanced (since the number of paroxysmal patients is about a quarter of 
the total number of persistent subjects), we have divided the patients into two different data sets: 40 patients (20 paroxysmal and 20 persistent) to train the classifier, and 146 patients (21 paroxysmal and 125 persistent) to be used as the test dataset. This dataset includes a huge variety of patients who are under different antiarrhythmic drugs, some who have undergone catheter ablation, some who present other comorbidities, and with different state of progression of the arrhythmia (including first AF episodes and recurrent ones). Thus, this dataset diversity is similar to the patients heterogeneity that clinicians must deal with in their daily activity. Clinical characteristics of the subjects included in the present study are shown in Table 1.

\section{Methods}

\subsection{Signal preprocessing}

The first step when processing the ECG signal was to remove the baseline and powerline noise. Then, we upsampled the signal to $1000 \mathrm{~Hz}$ so as to the $\mathrm{R}$ peak detection [26] and their alignment were more accurate for the subsequent QRST complex subtraction [27]. Next, we took advantage of the uncoupling of atrial and ventricular activities during AF, and we extracted atrial activity by suppressing the QRST complexes of the ECG signal using the method presented in [28], since the average beat subtraction is the most widely used method when single-lead information is available.

Thus, we canceled the ventricular activity of the ECG signal prior to processing the Fast Fourier Transform (FFT) [29]. Afterwards, the respective 
coefficients of sparse representations with respect to a Gabor frame calculated for FFT modulus and phase values are obtained, as it is detailed in the subsequent sections. Henceforth, for the sake of simplicity, we will refer to them as sparse coefficients.

Table 1: Statistical summaries of the database (n,\%). Hypertension was defined as a systolic blood pressure $\geq 140 \mathrm{mmHg}$, a diastolic blood pressure $\geq 90 \mathrm{mmHg}$, or if the patient was prescribed antihypertensive medication(s). Diabetes mellitus was defined as serum fasting glucose $\geq 7.0 \mathrm{mmol} / \mathrm{L}$ or on medications. Hypercholesterolemia was defined as cholesterol $\geq 6.4 \mathrm{mmol} / L$ or treatment with lipid-lowering drugs. Structural heart disease is defined as LV hypertrophy $>15 \mathrm{~mm}, L V E F<50 \%$, moderate or greater degrees of valvulopathy, prior myocardial infarction, significant coronary artery disease or the presence of primary myocardial diseases. AF: Atrial fibrillation. LV: left ventricle. Parox: paroxysmal, Pers: persistent, according to current clinical guidelines.

\begin{tabular}{lcccc}
\hline & $\begin{array}{c}\text { Parox. AF } \\
(\mathrm{n}=41)\end{array}$ & $\begin{array}{c}\text { Pers. AF } \\
(\mathrm{n}=145)\end{array}$ & $\begin{array}{c}\text { Overall } \\
(\mathrm{n}=186)\end{array}$ & p-value \\
\hline Age (mean, range) & $59(30-92)$ & $65(39-84)$ & $63(30-92)$ & 0.043 \\
Male (n,\%) & $22(54 \%)$ & $90(62 \%)$ & $112(60 \%)$ & 0.429 \\
Hypertension & $23(56 \%)$ & $91(63 \%)$ & $114(61 \%)$ & 0.554 \\
Diabetes & $5(12 \%)$ & $46(32 \%)$ & $51(27 \%)$ & 0.023 \\
Hypercholesterolemia & $12(29 \%)$ & $55(38 \%)$ & $67(36 \%)$ & 0.403 \\
Any structural heart disease & $12(29 \%)$ & $84(58 \%)$ & $96(52 \%)$ & 0.002 \\
Valvular heart disease & $7(17 \%)$ & $53(37 \%)$ & $59(32 \%)$ & 0.030 \\
Impaired LV function & $5(12 \%)$ & $34(23 \%)$ & $39(21 \%)$ & 0.174 \\
Previous electric cardioversion & $2(5 \%)$ & $16(11 \%)$ & $18(10 \%)$ & 0.380 \\
Previous AF ablation & $0(0 \%)$ & $11(8 \%)$ & $11(6 \%)$ & 0.149 \\
Left Atrium dilatation & $6(15 \%)$ & $48(33 \%)$ & $54(29 \%)$ & 0.035 \\
Antiarrhythmic drugs & $15(37 \%)$ & $51(35 \%)$ & $66(35 \%)$ & 1 \\
Betablockers & $14(34 \%)$ & $73(50 \%)$ & $87(47 \%)$ & 0.097 \\
Digoxin & $2(5 \%)$ & $22(15 \%)$ & $24(13 \%)$ & 0.141 \\
Calcium channel antagonists & $1(2 \%)$ & $10(7 \%)$ & $11(6 \%)$ & 0.488 \\
\hline
\end{tabular}



and $\|\cdot\|$ is the euclidean norm

A family of vectors $\left(\varphi_{j}\right)_{j=0}^{J-1}$ in $\mathbb{C}^{L}$ is called a frame for $\mathbb{C}^{L}$ if there exist constants $0<K_{1} \leq K_{2}$ such that

$$
K_{1}\|f\|^{2} \leq \sum_{j=0}^{J-1}\left|\left\langle f, \varphi_{j}\right\rangle\right|^{2} \leq K_{2}\|f\|^{2} \text { for all } f \in \mathbb{C}^{L}
$$

The numbers $\left\langle f, \varphi_{j}\right\rangle, 0 \leq j \leq J-1$, are called the frame coefficients of $f$. Associated to any family of vectors $\left(\varphi_{j}\right)_{j=0}^{J-1}$ in $\mathbb{C}^{L}$ one has the analysis operator

$$
A: \mathbb{C}^{L} \rightarrow \mathbb{C}^{J} \text { defined as } A(f)=\left(\left\langle f, \varphi_{j}\right\rangle\right)_{j=0}^{J-1}
$$

and its adjoint the synthesis operator

$$
A^{*}: \mathbb{C}^{J} \rightarrow \mathbb{C}^{L}, \quad A^{*}(\gamma)=\sum_{j=0}^{J-1} \gamma(j) \varphi_{j}, \quad \text { where } \gamma=[\gamma(0), \gamma(1), \ldots \gamma(J-1)]
$$

Hence, the family $\left(\varphi_{j}\right)_{j=0}^{J-1}$ is a frame for $\mathbb{C}^{L}$ if and only if $A$ is injective or equivalently, if and only if $A^{*}$ is surjective. Thus, $\left(\varphi_{j}\right)_{j=0}^{J-1}$ is a frame for $\mathbb{C}^{L}$ if and only if each signal vector $f$ in $\mathbb{C}^{L}$ can be expressed as a linear combination of vectors $\left\{\varphi_{0}, \varphi_{1}, \ldots, \varphi_{J-1}\right\}$. Frames in $\mathbb{C}^{L}$ consisting of $L$ elements are in 
fact bases. Frames with $J>L$ elements are called redundant. In the case that the family $\left(\varphi_{j}\right)_{j=0}^{J-1}$ is a frame for $\mathbb{C}^{L}$ the operator $A^{*} A$ is called the frame operator. It is a self-adjoint, positive and invertible operator. This means that each vector $f$ can be reconstructed from its frame coefficients.

For a discrete non-zero window $g \in \mathbb{C}^{L}$ and $0 \leq k, \ell \leq L-1$ we write $\left(\pi_{k, \ell} g\right)(n)=g(n-k) e^{-2 \pi i \ell n / L}$. Then, $\pi_{k, \ell} g$ represents a translation and modulation of the window $g$. The Gabor transform $V_{g}: \mathbb{C}^{L} \rightarrow \mathbb{C}^{L \times L}$ with respect to the window $g$ is the injective and linear map defined by

$$
V_{g} f(k, \ell)=\left\langle f, \pi_{k, \ell} g\right\rangle=\sum_{n=0}^{L-1} f(n) \overline{g(n-k)} e^{2 \pi i \ell n / L} .
$$

The Gabor system generated by the window $g$ and $\Lambda \subset\{0,1, \ldots, L-$ $1\} \times\{0,1, \ldots, L-1\}$ is the set of vectors $\left\{\pi_{k, \ell} g:(k, \ell) \in \Lambda\right\}$. If the Gabor system is a frame, we call it a Gabor frame.

A typical choice of $\Lambda$ is as follows: for $a, b \in \mathbb{N}$ and $N, M$ with $N a=$ $M b=L$ we let

$$
\Lambda:=\{(n a, m b): n=0, \ldots, N-1, m=0, \ldots, M-1\} .
$$

The parameters $a$ and $b$ represent time and frequency sampling intervals. In order to have a frame, $a b \leq L$. The case $a b=L$ is referred as the critically sampled Gabor transform and the case $a b<L$ yields an oversampled Gabor transform. For detailed information about finite frames see the book [30] and the references therein. 

sentations

$$
f=\sum_{m, n} \gamma(n, m) \pi_{n a, m b} g
$$

with the degree of freedom identified with the dimension of the null-space of $A^{*}$. We seek for the sparsest representation of a signal as a linear combination of the atoms of a Gabor frame. More precisely, for a signal $f \in \mathbb{C}^{L}$ and a Gabor frame $\left\{\pi_{n a, m b} g, n=0, \ldots, N-1, m=0, \ldots, M-1\right\}$ where $M N>>L$ we look for the coefficient vector $\gamma \in \mathbb{C}^{M \times N}$ solving the convex optimization problem

$$
\text { Minimize } \sum_{n, m}|\gamma(n, m)| \text {, subject to } f=\sum_{m, n} \gamma(n, m) \pi_{n a, m b} g \text {. }
$$

The implementation to obtain the sparse coefficients has been made by using the LTFAT toolbox. For detailed information about the methods see [31].

\subsection{Principal Component Analysis}

Principal Component Analysis (PCA) provides a new coordinate system such that the new axes point into the directions of highest variance of the data [32]. Each new variable (called principal component) is obtained as a 
linear combination of the original variables, so that each principal component is orthogonal to the rest. In this manner, redundant information can be suppressed and the number of features that feed the classifier can also be reduced.

To that end, each axis is chosen consecutively in the direction where the variance of the original data is maximum. These are the eigenvectors of the covariance matrix of the data, which correspond to the respective eigenvalues once they have been decreasingly ordered. Then, the new variables are obtained by projecting the original ones on the new axis.

We have performed PCA on the sparse coefficients in order to reduce the number of significant features by keeping most of its relevant information.

\subsection{Feature extraction}

Once the modulus and phase of the FFT input are processed to obtain the sparse coefficients, we apply PCA analysis to each subset after their linear normalization to the range $[0,1]$. This way, PCA helps to reduce the number of features to be considered. In addition, we also calculate the logarithm of the energy entropy for each group of the sparse coefficients, which is defined as:

$$
E(s)=\sum_{i} \log \left(s_{i}^{2}\right)
$$

with the convention $\log (0)=0$, where $s_{i}$ refers to each element of the representation of the sparse coefficients in the principal component space. These features will measure the degree of complexity of the sparse coefficients of 
the frame.

Below, the flowchart shown in Figure 1 depicts the steps of the proposed method, where the classifier is feeded with:

- Entropy of the sparse coefficients of the modulus of the FFT in the principal component space, $E_{m}$.

- Entropy of the sparse coefficients of the phase of the FFT in the principal component space, $E_{p}$.

- The first four principal components of the sparse coefficients of the modulus of the FFT input $S_{m_{k}}, k=1, \cdots, 4$.

- The first two principal components of the sparse coefficients of the phase of the FFT input $S_{p_{k}}, k=1,2$.

- Average of distance between $\mathrm{R}$ peaks in the ECG signal $R R_{\text {mean }}$.

Classifier details are described in Section 3.5.

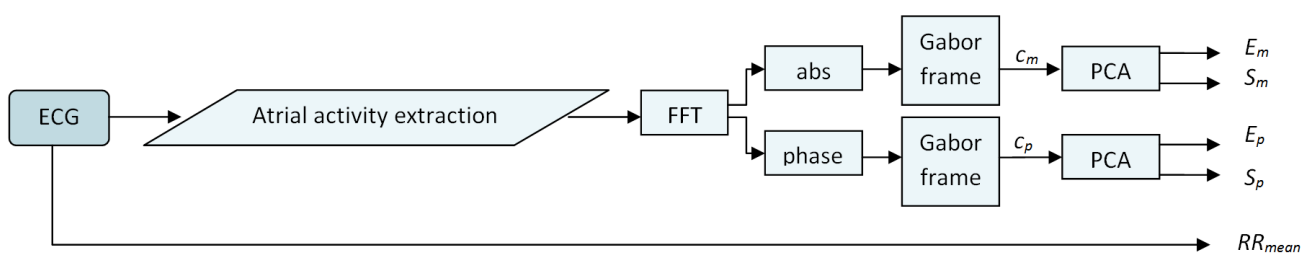

Figure 1: Flowchart of proposed features extraction method. 


\subsection{Classification}

We have used linear discriminant analysis (LDA) for classifying patients, which is an efficient and low computational cost method [33]. LDA's objective is to reduce the dimensionality of the data and also to preserve most of the class discriminatory information by means of a model which assumes that both classes are linearly separable. Thus, if we assume that patients are classified into two classes, Fisher's linear discriminant [34] pursues to obtain the optimal hyperplane that maximises the separability of the feature vectors $x$. In order to find it, it is necessary to define a measure of separation between the projections, which should maximise the differences between the means projected on the hyperplane while also minimise the scatter (or variance) within classes.

In this study the LDA classifier was trained with 20 paroxysmal and 20 persistent AF episode signals, which were chosen by clinicians as those corresponding to patients who behave as 'clinical models' for each class (paroxysmal and persistent), in which AF patients are sorted according to current clinical guidelines. Given the unbalanced number of paroxysmal and persistent AF subjects that form the population under study, the use of jackknifing or leaving-one-out techniques was not a suitable option, since they produced biased results. We have carried out bootstrap analysis [35] with different number of training samples in order to choose their optimal size. It was observed that performances progressively increased as long as the number of samples used to train the classifier grew up to 40 training samples, meanwhile 
thereafter there was little or no-significant average increment in performance figures. Thus, we chose to train our classifier with 20 paroxysmal and 20 persistent subjects.

\section{Results}

\subsection{Performance measures}

Sensitivity and specificity are defined as the ratio of paroxysmal or persistent AF patients correctly classified to the total number of paroxysmal or persistent patients, respectively:

$$
\begin{aligned}
& \text { Sensitivity }=\frac{T P_{\text {paroxysmal }}}{T P_{\text {paroxysmal }}+F P_{\text {persistent }}} \\
& \text { Specificity }=\frac{T P_{\text {persistent }}}{T P_{\text {persistent }}+F P_{\text {paroxysmal }}}
\end{aligned}
$$

where TP (true positives) refers to the number of patients correctly classified according to its AF subtype, and FP (false positives) is the number of paroxysmal or persistent patients misclassified.

In addition, global accuracy is measured by means of average accuracy (4), in order to eliminate the influence of the unbalanced test dataset with the number of persistent patients being about 4 times larger than the number of paroxysmal subjects:

$$
\text { Average accuracy }=\frac{\text { Sensitivity }+ \text { Specificity }}{2}
$$


Classification performance has also been measured by means of the receiver operating characteristic (ROC) curve, which plots sensitivity against (1-specificity), and by the area under the ROC curve.

\subsection{Experimental results}

This section contains the classification performances obtained for the proposed method (first row of Table 2), and a comparison with several recent references of the state-of-the-art that address the analysis of spontaneous self-termination AF, or sustained and permanent AF.

In the proposed method, both sparse representations of modulus and phase information were with respect to Gabor frames, whose parameters have been iteratively adjusted, in order to maximise the average accuracy when classifying the training dataset. Length of each analysed signal was 4096 samples, whereas the number of shifts $N$ and the number of modulations $M$ were experimentally set in both frames to 64 and 256, respectively.

Table 2 shows that the proposed method outperforms other recent references (addressed to study selt-terminating and sustained AF) when classifying the patients included in our database, obtaining about $89 \%$ of average accuracy, and sensitivity and specificity performances about 95\% and $82 \%$, respectively. These values represent an improvement of global accuracy about 5-10\% with respect to the other two works that perform best [10]-[13]. These results can also be observed in Figure 2, which displays the Receiver Operating Characteristic (ROC) curve for the proposed classification method, as 
well as the respective ROC curves for other relevant references. The values of the areas under convergence (AUC) for each ROC curve are also detailed, which additionally support results indicated in Table 2 .

Nevertheless, this comparison should be carefully evaluated, since the clinical AF classification problem is not equivalent, and clinical databases are different indeed. Discussion about this remark will be enlarged in Section 5 .

Table 2: Classification results for the test dataset (146 patients: 21 paroxysmal and 125 persistent). LDA classifier has been trained with 20 paroxysmal and 20 persistent AF patients. Results for the proposed method are also compared on the same test dataset with relevant references of the state-of-the-art which analyse AF.

\begin{tabular}{cccc}
\hline Features & Sensitivity & Specificity & $\begin{array}{c}\text { Average } \\
\text { accuracy }\end{array}$ \\
\hline Proposed & 0.9524 & 0.8240 & 0.8882 \\
\hline Dominant frequency of AA [7] & 0.2857 & 0.7440 & 0.5149 \\
\hline $\begin{array}{c}\text { Dominant frequency of AA, heart rate } \\
\text { distance between R peaks [13] }\end{array}$ & 1 & 0.672 & 0.836 \\
\hline Sample entropy [15] & 0.3810 & 0.6480 & 0.5145 \\
\hline Phase variations of GFT [10] & 0.8095 & 0.7840 & 0.7877 \\
\hline
\end{tabular}




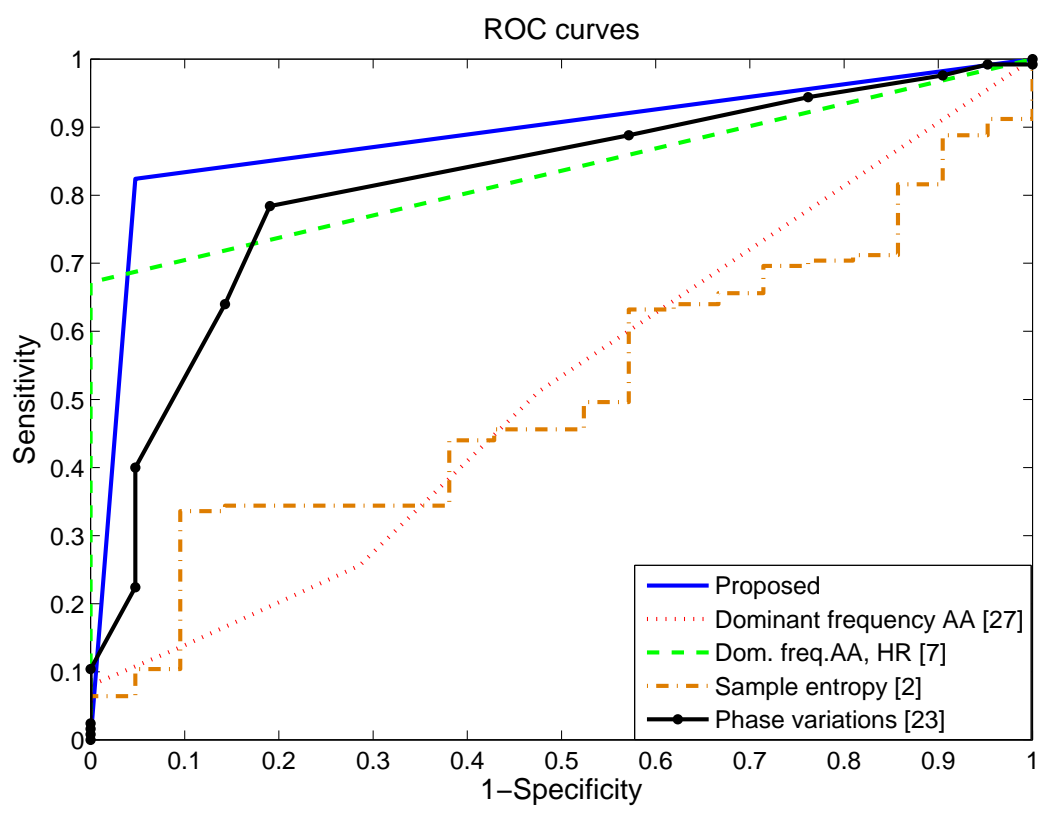

Figure 2: Receiver operating characteristic curves for the proposed method and several recent references. ROC curves have been obtained for the test dataset (146 patients: 21 paroxysmal, 125 persistent). Corresponding areas under ROC curves are: 0.8953 for the proposed method, 0.5168 for [7], 0.8360 for [13], 0.5109 for [15], and 0.8154 for [10]. 


\section{Discussion}

In this paper, classification of paroxysmal and persistent AF episodes according to current clinical guidelines has been studied by means of frame analysis. Good performances have been obtained, which may be of great utility in order to provide clinical assessment to help clinicians to choose the most suitable and effective treatment for patients under AF.

The lack of access to Holter recordings of patients included in the retrospective study was a major drawback, as the common duration of ECG recordings stored at the tertiary centre where the research was conducted was 5 seconds of length. Therefore, this study aimed for classification of AF episodes by processing very short ECG segments. Although this difficulty was overcome and good results were obtained (details on Table 2 and Figure 2 ), even including patients with multiple pathologies and different state of progression of the arrhythmia (Table 1), the comparison with other methods which have studied AF termination should be carefully evaluated by two main reasons. First, because the clinical problem presented in this paper is not exactly the same as the one proposed in other references, such as $[13,15,14]$ : most of them study spontaneous self-termination of AF versus sustained AF for 24 hours based on Physionet AF Termination and Long-Term databases $[6,7]$ whereas we have proposed the classification between paroxysmal and persistent AF episodes according to current guidelines $[3,4,5]$. Second, some references in Table 2 may not perform optimally and offer poorer results due to the short length of the recordings used in this retrospective study. Doubt- 
less, they would have performed more properly if we had had long recordings on the same dataset, which unfortunately is not possible currently.

Another limitation of the cohort of consecutive unselected patients was that AF subtypes were unbalanced in number: there were about four times more persistent than paroxysmal AF patients. Moreover, it is important to remark that the clinical cost of misclassification is higher for paroxysmal patients than for those persistent. This is due to the fact that an early paroxysmal atrial fibrillation detection allows a preventive AF treatment against recurrence, such as pulmonary vein isolation, which has been proved to be one of the best options to stop AF progression. Despite the unbalanced dataset, the proposed method obtains not only unbiased results (89\% of average accuracy), but also about $95 \%$ of paroxysmal subjects properly classified.

Regarding feature extraction, along the study we tried to characterize the AF subtypes features by using several types of frame, but Gabor frames were the ones which provided the best classification results. For AF analysis and spontaneous self-termination prediction, most of the state-of-the-art references have hitherto included Fourier analysis, time-frequency analysis or even non-linear measures of the atrial activity. As previous works have pointed out [10], patients suffering from a paroxysmal AF episode present lower phase variations when studying both the time and frequency domains of the ECG signal. From a physiological point of view, this is explained by the fact that paroxysmal atrial fibrillation patients have lower levels of atrial fibrosis when compared with persistent atrial fibrillation patients. These lower 
levels of fibrosis are translated into a faster and more homogeneous electrical conduction in the atrium of patients with paroxysmal atrial fibrillation which may be expressed by lower phase variations.

This idea is also related with the results presented in this work. The representation of a signal obtained by the different time-frequency transforms is related with the concrete representation that can be obtained by means of the frame coefficients of the signal with respect to a particular frame. However, if the frame is highly redundant (as it is proposed in this paper), we can obtain many representations of the signal as linear combinations of the frame atoms. Thus, the choice of the sparsest representation fulfilled in this paper has allowed us to improve classification performances by choosing the most suitable representation in that frame. In this manner, presented results reveal that Gabor frames are an efficient and excellent alternative to the aforementioned tools for signal analysis. This different point of view has been successfully applied to ECG compression [20, 21] and atrial activity extraction [24] but, to our knowledge, this is the first attempt to use it for early differentiation of AF episodes, with a significant improvement with respect to previous references that have addressed this classification problem.

With regard to clinical implications, it is highly significant to notice the importance of an early differentiation of the nature of the AF episode to which clinicians have to face with. The standard therapy for paroxysmal AF patients who present recurrences despite the antiarrhythmic drug treatment is the catheter ablation procedure implying pulmonary vein isolation. In this 
context, ablation offers success rates at one year of $70-80 \%$. On the contrary, success rates of pulmonary vein isolation in persistent AF patients are much worse, around $45-60 \%$ at best. The efficacy of ablation in persistent AF patients can be improved by performing additional ablation lines (in the left atrial roof, mitral isthmus or posterior wall), or by ablation of complex fractional atrial electrograms (CFAEs) besides the pulmonary vein isolation. This is why the early knowledge of the type of the arrhythmia of each patient may have vital therapeutic implications, such as the modification and individualization of the therapy to be administered to each patient. Furthermore, the proposed method achieves this goal using a widely available resource in daily clinical practice (the ECG), and with no need of any further exploration to the ones which are routinely carried out in these patients.

\section{Conclusions}

A new classification method of paroxysmal and persistent AF episodes has been presented. It is based on extracting the coefficients of a sparse representation with respect to a Gabor frame, which have been obtained from the frequency spectrum of the atrial activity of short ECG segments. Then, extracted features pass through an LDA classifier, which has been trained to maximise both sensitivity and specificity measures. Good results on real ECG recordings are achieved, which is important to remark as a value-add of the study, specially taking into account that they are obtained on a cohort of patients who present different states of AF progression, are under different 
antiarrhythmic treatments, and some of which present multiple pathologies.

Future work will focus on enlarging the dataset and analyse results on a prospective study with long recordings.

\section{Acknowledgements}

This work was supported by Generalitat Valenciana under grants PrometeoII/2013/013, ACOMP/2015/186, and by MINECO under grant MTM201343540-P.

\section{Competing interests}

None declared

\section{Ethical approval}

Ethical approval was obtained by the participating centres: Hospital Universitari i Politècnic La Fe and Instituto de Investigación Sanitaria La Fe. Patients also signed an agreement allowing to use their data for clinical studies.

\section{References}

[1] Go A, Hylek E, Phillips K, Chang Y, Henault L, Selby J, et al. Prevalence of diagnosed atrial fibrillation in adults: national implications for rhythm management and stroke prevention: the AnTicoagulation and 
Risk Factors in Atrial Fibrillation (ATRIA) Study. The Journal of the American Medican Association 2011;285(18):2370-2375.

[2] Sörnmo L, Laguna P. Bioelectrical Signal Processing in Cardiac and Neurological Applications. Elsevier Academic Press; 2005.

[3] Fuster V, Rydén L, Cannom D, Crijns H, Curtis A, Ellenbogen K, et al. ACC/AHA/ESC 2006 Guidelines for the Management of Patients with Atrial Fibrillation: a report of the American College of Cardiology/American Heart Association Task Force on Practice Guidelines and the European Society of Cardiology Committee for Practice Guidelines (Writing Committee to Revise the 2001 Guidelines for the Management of Patients With Atrial Fibrillation): developed in collaboration with the European Heart Rhythm Association and the Heart Rhythm Society. Circulation 2006;114(7):e257-e354.

[4] Wann L, Curtis A, January C, Ellenbogen K, Lowe J, Estes N, et al. 2011 $\mathrm{ACCF} / \mathrm{AHA} / \mathrm{HRS}$ focused update on the management of patients with atrial fibrillation (updating the 2006 guideline): a report of the American College of Cardiology Foundation/American Heart Association Task Force on Practice Guidelines. Circulation 2011;123(1):104-123.

[5] January C, Wann L, Alpert J, Calkins H, Cigarroa J, Cleveland J, et al. 2014 AHA/ACC/HRS Guideline for the Management of Patients With Atrial Fibrillation. A Report of the American College of Cardi- 
ology/American Heart Association Task Force on Practice Guidelines and the Heart Rhythm Society. J Am Coll Cardiol 2014;64(21):e1-e76.

[6] Moody G. Spontaneous termination of atrial fibrillation: A challenge from PhysioNet and computers in cardiology. Computers in Cardiology $2004 ; 31: 101-104$.

[7] Petrutiu S, Sahakian A, Swiryn S. Abrupt changes in fibrilatory wave characteristics at the termination of paroxysmal atrial fibrillation in humans. Europace 2007;9(7):466-470.

[8] Goya-Esteban R, Sandberg F, Barquero-Perez O, Garcia-Alberola A, Sörnmo L, Rojo-Alvarez J. Long-term characterization of persistent atrial fibrillation: wave morphology, frequency, and irregularity analysis. Med Biol Eng Comput 2014;52(12):1053-1060.

[9] Ortigosa N, Cano O, Ayala G, Galbis A, Fernández C. Atrial fibrillation subtypes classification using the General Fourier-family Transform. Med Eng Phys 2014;36(4):554-560.

[10] Ortigosa N, Fernández C, Galbis A, Cano O. Phase information of time-frequency transforms as a key feature for classification of atrial fibrillation episodes. Physiol Meas 2015;36(3):409-424.

[11] Sandberg F, Stridh M, Sörnmo L. Frequency Tracking of Atrial Fibrillation Using Hidden Markov Models. IEEE Trans Biomed Eng $2008 ; 55(2): 502-511$. 
[12] Nilsson F, Stridh M, Bollmann A, Sörnmo L. Predicting spontaneous termination of atrial fibrillation using the surface ECG. Med Eng Phys 2006;28(8):802-808.

[13] Chiarugi F, Varanini M, Cantini F, Conforti F, Vrouchos G. Non invasive ECG as a Tool for Predicting Termination of Paroxysmal Atrial Fibrillation. IEEE Trans Biomed Eng 2007;54(8):1399-1406.

[14] Alcaraz R, Rieta J. The application of nonlinear metrics to assess organization differences in short recordings of paroxysmal and persistent atrial fibrillation. Physiol Meas 2010;31(1):115-130.

[15] Alcaraz R, Rieta J. Sample entropy of the main atrial wave predicts spontaneous termination of paroxysmal atrial fibrillation. Med Eng Phys 2009;31(8):917-922.

[16] Alcaraz R, Sandberg F, Sörnmo L, Rieta J. Application of Frequency and Sample Entropy to Discriminate Long-Term Recordings of Paroxysmal and Persistent Atrial Fibrillation. Conf. Proc. IEEE Eng. Med. Biol. Soc.; 2010, p. 4558-4561.

[17] Cervigón R, Moreno J, Reilly R, Millet J, Pérez-Villacastín J, Castells F. Entropy measurements in paroxysmal and persistent atrial fibrillation. Physiol Meas 2010;31:1011-1020.

[18] Sahoo S, Lu W, Teddy S, Kim D, Feng M. Detection of Atrial fibrillation 
from non-episodic ECG data: a review of methods. Conf. Proc. IEEE Eng. Med. Biol. Soc.; 2011, p. 4992-4995.

[19] Seungjae L, Jun L, Chou P. A new approach to compressing ECG signals with trained overcomplete dictionary. 4th Int. Conf. on Wireless Mobile Communication and Healthcare; 2014, p. 83-86.

[20] Fira M, Goras L, Barabasa C, Cleju N. On ECG Compressed Sensing using Specific Overcomplete Dictionaries. Adv Electr Comput En $2010 ; 10(4): 23-28$.

[21] Skretting K, Engan K, Husøy J. ECG compression using signal dependent frames and matching pursuit. IEEE Int. Conf. on Acoustics, Speech and Signal Processing; 2005, p. 585-588.

[22] Pantelopoulos A, Bourbakis N. ECG Beat Classification Using Optimal Projections in Overcomplete Dictionaries . IEEE Int. Conf. on Tools with Artificial Intelligence; 2011, p. 1099-1105.

[23] Mailhe B, Gribonval R, Bimbot F, Lemay M, Vandergheynst P, Vesin J. Dictionary learning for the sparse modelling of atrial fibrillation in ECG signals. IEEE Int. Conf. on Acoustics, Speech and Signal Processing; 2009, p. 465-468.

[24] Divorra-Escoda O, Granai L, Lemay M, Molinero-Hernandez J, Vandergheynst P, Vesin J. Ventricular and Atrial Activity Estimation 
Through Sparse ECG Signal Decompositions. IEEE Int. Conf. on Acoustics, Speech and Signal Processing; 2006, p. II1060-II1063.

[25] Ortigosa N, Giménez V. Raw data extraction from electrocardiograms with Portable Document Format. Comput Meth Programs Biomed 2014;113(1):284-289.

[26] Pan J, Tompkins W. A real-time QRS detection algorithm. IEEE Trans Biomed Eng 1985;32(3):230-236.

[27] Bollmann A, Husser D, Mainardi L, Lombardi F, Langley P, Murray A, et al. Analysis of surface electrocardiograms in atrial fibrillation: techniques, research, and clinical applications. Europace 2006;8(11):911-926.

[28] Alcaraz R, Rieta J. Adaptive singular value cancellation of ventricular activity in single-lead atrial fibrillation electrocardiograms. Physiol Meas 2008;29(12):1351-1369.

[29] Cooley J, Tukey J. An Algorithm for the Machine Computation of the Complex Fourier Series. Math Comput 1965;19(90):297-301.

[30] Casazza P, Kutyniok G. Finite frames: Theory and applications. Birkhäusser; 2010.

[31] Søndergaard P, Torrésani B, Balazs P. The Linear Time Frequency Analysis Toolbox. Int J Wavelets Multiresolut Inf Process 2012;10(4):27, ID 1250032 . 
492

494

495

[32] Jolliffe I. Principal Component Analysis. Springer; 2002.

[33] Duda R, Hart P, Stork D. Pattern Classification. Wiley Interscience; 2000.

[34] McLachlan G. Discriminant Analysis and Statistical Pattern Recognition. Wiley Interscience; 2004.

[35] Efron B. Bootstrap methods: another look at the jackknife. The Annals of Statistics 1979;7(1):1-26. 